\title{
Flann O’Brien / Brian Ó Nualláin : portraits linguistiques d'un provocateur irlandais entre identité collective et idiosyncrasie ludique
}

Flann O'Brien/Brian Ó Nualláin: linguistic portraits of an Irish agitator between collective identity and ludic idiosyncrasy

\section{Thierry Robin}

\section{OpenEdition \\ Journals}

Édition électronique

URL : https://journals.openedition.org//bl/1063

DOI : $10.4000 / \mathrm{lbl} .1063$

ISSN : 2727-9383

Éditeur

Université de Bretagne Occidentale - UBO

\section{Édition imprimée}

Date de publication : 1 juin 2015

Pagination : 119-137

ISBN : 979-10-92331-16-5

ISSN : $1270-2412$

\section{Référence électronique}

Thierry Robin, «Flann O’Brien / Brian Ó Nualláin : portraits linguistiques d'un provocateur irlandais entre identité collective et idiosyncrasie ludique », La Bretagne Linguistique [En ligne], 19 | 2015, mis en ligne le 01 mai 2021, consulté le 22 mai 2021. URL : http://journals.openedition.org//bl/1063 ; DOI : https://doi.org/10.4000/lbl.1063

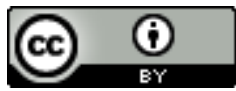

La Bretagne Linguistique est mise à disposition selon les termes de la Licence Creative Commons Attribution 4.0 International. 


\section{Flann O’Brien/Brian Ó Nualláin : portraits linguistiques d'un provocateur irlandais entre identité collective et idiosyncrasie ludique}

\section{"B} rian O’Nolan", "Count O'Blather", "Brother Barnabas", "Myles na gCopaleen", "George Knowall”, “John James Doe", "An Broc"1, "Flann O'Brien"... De prime abord, au vu de cette multiplicité de noms de plume, l'œuvre de Flann O'Brien semble clairement poser le problème de l'identité, et de son expression plurielle à travers le langage. Du reste, O'Brien demeure un brillant touche-à-tout littéraire. On citera évidemment ses romans At SwimTwo-Birds (1939), The Poor Mouth (1973, 1941 pour l'original en gaélique), The Third Policeman (1967, son chef-d'œuvre posthume), pour ne citer qu'eux. Mais O'Brien fut aussi dramaturge, nouvelliste, chroniqueur au Irish Times par sa rubrique intitulée An cruiskeen Lawn de 1940 à 1966, essayiste, critique, poète parodique... Le langage apparaît chez lui dans toutes ses dimensions, révélant ses forces et failles en tant qu'outil de création, ainsi que toute son ambivalence : à la fois source de sens et de repères et labyrinthe de

* Maître de conférences d'anglais, HCTI-CEIMA (EA 4249), UBO/ueb.

1. An Broc (proche de «ar broc'h» en breton) veut évidemment dire littéralement «le blaireau». 
faux-semblants, de faux-fuyants où l'on se perd dans les chimères de la représentation. Il est un miroir de la réalité simultanément exigeant et déformant. O'Brien s'engouffre d'ailleurs avec vigueur et esprit dans les failles qui séparent le réel de sa représentation, insistant sur un certain nombre d'apories reprises et expliquées par un penseur contemporain tel que Clément Rosset. Cette problématique identitaire voire ontologique alliée à celle du sens à accorder au langage est à rapprocher des préoccupations qui gisent au cœur d'un courant de pensée, ou plutôt d'un véritable éventail de mouvements critiques, des plus prolifiques depuis le début de la seconde moitié du $X^{\mathrm{e}}$ siècle : le postmodernisme. En effet, l'œuvre d'O'Brien, aux allures de boîte de Pandore, bien qu'en grande partie éclipsée par l'importance titanesque du monument joycien, semble avoir particulièrement séduit la critique postmoderne de Theo d'Haen à Keith Hopper. Mais au-delà des querelles théoriques portant sur les frontières conceptuelles poreuses entre modernisme, post-modernisme voire néo-modernisme, O'Brien permet de mieux penser, articuler le local et l'universel, le provisoire et l'atemporel, le fictif et le réel. Son œuvre écrite en partie en gaélique, et bien sûr en anglais, questionne en profondeur les concepts d'identité, de particularisme ou d'universalité et ce avec une originalité langagière et une réflexion linguistique ironique portant tout autant sur l'anglais que le gaélique. O'Brien situe tous ses écrits ou presque en Irlande. Son humour, ses personnages, ses intrigues, ses tabous et réjouissances liées à la religion ou à l'alcool - voire les deux mêlés comme dans The Hard Life -, ses repères historiques (depuis l'âge d'or supposé des Gaëls) jusqu'à la famine ou la politique nationaliste irlandaise, dans un contexte colonial, ou celui de l'État libre d'Irlande ou même de la République après 1949, renvoient tous à cette contrée tantôt réelle tantôt rêvée voire cauchemardée. Pour ce qui est du gaélique, on songe évidemment à An Béal Bocht, traduit tardivement par Patrick C. Power en The Poor Mouth et à de nombreuses rubriques, surtout au début de sa carrière au Irish Times, ainsi qu'à des nouvelles peu diffusées mais assez amusantes, dont voici quelques titres et synopsis assez révélateurs de l'importance occupée par la langue en tant que système linguistique ou outil politique de domination voire lieu de recherche archéologique. On remarque que l'abord est parodique et inventif : 
«- 'Díoghaltais ar Ghallaibh 'sa Bhliain 2032!' ["Revanche sur l'anglais en l'an 2032!"]. The Irish Press, 18 janvier 1932. (Synopsis de Jack Fennell : l'intrigue futuriste se situe dans une Irlande unie parlant le gaélique. Un Irlandais bilingue enseigne à un touriste anglais une expression irlandaise prétendant qu'il pourra ainsi demander son chemin, il s'agit en fait d'un chapelet d'obscénités qui plonge l'Anglais dans bien des tracas quand il le récite.)

— 'Teacht Agus Imtheacht Sheáin Bhuidhe' ["L'arrivée et le depart de John Bull"]. The Irish Press, 13 juin 1932. (Synopsis de Jack Fennell : dans un avenir lointain, alors que l'irlandais est la langue dominante et que l'anglais s'est éteint; John Bull fait des recherches pour 'Connradh an Bhéarla' et vient en Irlande en quête de fragments de vocabulaire anglais) ${ }^{2}$. "

On voit dans ces deux nouvelles, l'intérêt que porte $\mathrm{O}^{\prime}$ Brien à la langue. Dans les deux cas, O'Brien s'amuse à renverser l'ordre des choses : l'anglais devient curiosité archéologique, minoritaire, le gaélique devient dominant, dans une vision ironique du futur où la Gaelic League laisse la place à 'Connradh an Bhéarla', son pendant saxon. La même approche ludique caractérise O’Brien quant au langage en tant que système réflexif imparfait, décalé par rapport au réel, quand il reprend à son compte l'orthographe shavienne ${ }^{3} \mathrm{du}$ simple mot fish qu'il épèle alors "ghoti" en reprenant le "gh" de "laugh", le "o" de "women" et le "ti" de "nation" 4 . Ou encore quand O'Brien s'amuse à transcrire l'anglais (quand il ne s'agit pas plaisamment du français ${ }^{5}$ ) avec une orthographe phonétique empruntée au gaélique comme dans le passage suivant de The Best of Myles :

«Sheán Buidhe: Fbhait ár iúr méin traighing thú sae, Sairdint?

Sairdint Tharbhaigh: Aigh tink dae ár tócuing abamht a bhuman cóld Agnes, a biútiful accomplas eigh supós. »

2. http://www.univie.ac.at/flannobrien2011/bibliography.html, accès le 28 novembre 2013. Je traduis.

3. D'après George Bernard Shaw.

4. Anecdote citée de Thomas SHEA, Flann O'Brien's Exorbitant Novels, Bucknell University Press, Londres et Toronto, Associated University Press, 1992, p. 8687.

5. Myles na GCopaleen, The Best of Myles, (Kevin O’Nolan dir.), MacGibbon \& Kee, Londres, 1968. Londres, Flamingo, 1993, p. 271. 
Ce qui correspond à :

«John Bull: What are your men trying to say, Sergeant?

Sergeant Tharbhaigh: I think they are talking about a woman called Agnes, a beautiful accomplice I suppose ${ }^{6}$. »

Ce à quoi procède $\mathrm{O}^{\prime}$ Brien par son style singulier et son traitement iconoclaste du langage est une déconstruction délibérée de l'identité irlandaise, notamment quand celle-ci se cristallise autour de clichés. Ce faisant, O'Brien présente les singularités mais aussi les contradictions ainsi que les aspects universels de cette identité. Je procéderai en quatre temps pour analyser cette déconstruction, ou encore déconstruire cette déconstruction. Tout d'abord, j'aborderai la notion de cliché, puis je tenterai d'identifier les ancrages identitaires linguistiques présents dans l'œuvre. Après un détour par l'idée de nationalisme, dans un quatrième et dernier temps, je me pencherai sur le gaélique, langue qu'O'Brien parlait à la perfection, mais dont il percevait à la fois la beauté poétique, la valeur historique mais aussi - et peut-être surtout pour le satiriste qu'il était - les angles morts, les limites en ces temps de modernité techniciste croissante $\mathrm{du} \mathrm{XX}^{\mathrm{e}}$ siècle.

\section{Stéréotypes... le plaisir de les transgresser tout en les réaffirmant paradoxalement...}

«Expliquez ce que cela fait d'être irlandais. Établissez l'âge auquel vous avez compris que vous étiez irlandais. Quand vous êtes-vous bagarré pour la première fois? À quel âge ? À quel âge avez-vous produit votre premier trait d'esprit irlandais ? À quel âge êtes-vous devenu un ivrogne ? Dites-moi tout s'il vous plait, car il n'y aura pas de remède tant que le fond pathologique n'aura pas été exploré. [...] C'est dans votre intérêt de tout me dire. Rappelez-vous que moi aussi j'ai été irlandais. Aujourd'hui je suis guéri. Je ne suis plus irlandais. Je suis un simple être humain. Je me suis guéri après maintes années de souffrances ${ }^{7}$. »

6. Ibid., p. 262.

7. Myles na GCopaleEn, Flann O'Brien At WarMyles na gCopaleen 1940-1945, (John Wyse Jackson dir.), Londres, Duckworth \& Co Ltd, 1999, p. 105, je traduis. 
Cette apostrophe questionne la litanie des clichés sur l'identité irlandaise. Elle les liste un à un : l'Irlandais est bagarreur, spirituel, alcoolique... O'Brien joue sur ces clichés et assimile l'irlandité à une maladie, ses caractéristiques à des symptômes dont il est évidemment infiniment souhaitable de se débarrasser. Ce passage est précisément symptomatique de la pensée d'O'Brien car il construit et déconstruit ironiquement le discours identitaire. Dans un premier temps, il dissèque les clichés qui composent l'imagerie d'Épinal sur les Irlandais, les réfutant du même coup implicitement. Mais ce qu'il déconstruit simultanément c'est le préjugé anti-irlandais, assimilant l'irlandité à une tare, une pathologie, une caricature grotesque correspondant peu ou prou au «Stage Irishman» - si visible chez un Richard Brinsley Sheridan par exemple. Cette ambivalence, cette polyphonie ironique, ces deux voix que l'on entend en même temps dans le récit d'O'Brien/Myles na gCopaleen font bien de lui un auteur de la construction/déconstruction ${ }^{8}$ postmoderne du discours. Cette postmodernité peut sembler apatride, universelle et décontextualisée, car parodie et satire relativisent tout ancrage localisé, vu comme folklorique et arbitraire. L'écrivain du non-sens n'a que faire de l'idée de nationalisme, d'idéalisation romantique de l'Irlande. On peut rejoindre Monique Gallagher lorsqu'elle affirme :

« À l'inverse de Joyce qui enrichit chaque épisode de significations multiples, O'Brien dépouille souvent ses histoires de toute signification : comme tous les épisodes qui ne doivent leur existence qu'à l'introduction du jeu de mots qui les conclut, le propos n'est souvent que le résultat d'une mécanique tournant à vide. L'anecdote ne révèle rien. Ainsi ce qu'il y a d'irresponsable dans les choix d'O'Brien, dans les jeux auxquels il se livre, et qui fait parfois penser qu'il se moque du lecteur, appartient en fait à la thématique de l'absurde $[\ldots]^{9}$.»

Absurde irlandais et «nonsense» anglais font ici bon ménage et convergent même. Monique Gallagher renvoie aux jeux de mots

8. L'un ne va pas sans l'autre. Dans The Best of Myles, op. cit., il va jusqu'à affirmer : «[...] Ireland is a vast construction job [...]», (p. 322). Ce « construction job », ce chantier, est éminemment linguistique et réflexif.

9. Monique Gallagher, Flann O’Brien, Myles na Gopaleen et les autres, Arras, Presses Universitaires de Lille III, (P.U. du Septentrion), 1998, p. 216-217. 
présents dans les titres de la série « Keats et Chapman », textes courts et amusants dont la logique à rebours tourne volontairement à vide. Pourtant, surprenant paradoxe, on trouve chez Flann O'Brien une écriture particulièrement imprégnée de ses racines irlandaises. C'est ce qui fait du reste toute la valeur d'O'Brien : à la fois profondément singulier et irlandais mais ne succombant jamais aux stéréotypes de la pire idéologie nationaliste étriquée. On se rappellera au passage qu'O'Brien tenait les clichés phraséologiques en horreur, comme en témoigne son "Catechism of Cliché», reproduit en grande partie dans l'anthologie de chroniques The Best of Myles ${ }^{10}$.

\section{Irlandismes et ancrages identitaires}

L'irlandité est au cour explicite du texte d'O'Brien, (contrairement à un Beckett par exemple), qui recourt à la parodie pour la déconstruire. Les mythes et légendes celtiques sont utilisés, recyclés dans le roman At Swim-Two-Birds, où le géant Finn Mac Cool, la razzia des vaches de Cooley ${ }^{11}$, ou encore la folie du roi légendaire Sweeney ${ }^{12}$ (Buile Shuibhne en gaélique), sont des motifs récurrents. La vie quotidienne à Dublin du pub à l'université pour le même roman At Swim ou bien dans le Gaeltacht pour An Béal Bocht $t^{13}$ nourrissent l'imaginaire d'O'Brien qui réécrit pour l'occasion le voyage de Maeldoon au chapitre 8 dans Le Pleure-Misère. La langue qu'il utilise témoigne ainsi de cette prégnance insulaire. Quand O'Brien n'utilise pas le gaélique directement comme dans An Béal Bocht, son anglais correspond assez bien à ce que l'on appelle le HibernoEnglish, ou anglais d'Irlande. Cela se traduit parfois par un calque phonétique délibéré, souvent surjoué, du traditionnel "brogue" ou accent irlandais. «Think» dans la citation déjà mentionnée mêlant les orthographes anglaise et gaélique, se prononce alors *«tink» sans

10. The Best of Myles, op. cit., p. 201-227.

11. Flann O'Brien, At Swim-Two-Birds, Londres, Longmans Green, 1939, rééd. Londres, Penguin Books, 1967, p. 18.

12. Ibid., p. 88-91.

13. Myles NA GCopaleEn, An Béal Bocht, Dublin, An Preas Naisiunta, 1941, traduit en anglais par Patrick C. Power, The Poor Mouth, Londres, Hart-Davis, MacGibbon Ltd, 1973, rééd. Londres, Flamingo, 1993, 128 p. (Traduit en français par Alain Le Berre et André Verrier sous le titre Le Pleure-misère, Paris, Le Tout sur le Tout, 1984.) 
« $\mathrm{h} »$. L'Amérique dans The Third Policeman ne s'écrit pas comme en anglais standard "America», mais * «Amurikey» ${ }^{14}$. Le démon ne s'épèle pas «devil» mais $*$ «divil ${ }^{15}$. L'adjectif emblématique «queer» s'altère en * «quare» dans At Swim, révélant le décalage existant entre le système de diphtongues R.P. ${ }^{16}$ et celui prévalant en République d'Irlande. Ainsi «decent» est souvent épelé «dacent» pour les « Plain People of Ireland» dans The Best of Myles, «easy» ${ }^{*}\left\langle\right.$ aisy $»{ }^{17}$. Cette mimésis dialectale se retrouve tout particulièrement dans le roman The Third Policeman, le chef-d'œuvre d'O'Brien, quand les policiers parlent. Mais une pièce assez courte comme Faustus Kelly (1943) le montre bien sans pour autant sombrer dans une déformation systématique ennuyeuse : «Decent» devient à nouveau «dacent» comme «Jesus» devient * «Jayzus». «Right» devient * «roight». «Certain» devient «sartain», «Certainly» : «sartinly». Le th-sonore devient un « $d$-» à la manière dont le th-sourd devient un $t$-. «That's right », s'épèle alors « dat's roight $»^{18}$.

Mais cette irlandisation phonétique de la langue ne se cantonne pas à la transcription superficielle d'un accent dans les mots, elle affecte aussi les structures et la grammaire du texte. L'article défini s'emploie de façon assez irlandaise même quand l'indéfini paraîtrait devoir s'imposer en anglais standard. Le défini prend alors une valeur archétypique somme toute assez rare dans les autres variétés de l'anglais. Ainsi Brinsley, l'ami du narrateur étudiant dans At Swim déclare à plusieurs reprises : "By God you're the queer bloody man ${ }^{19}$. " Le système des temps grammaticaux est modifié sous l'influence plus ou moins directe du substrat celtophone. Le passé immédiat dont la forme canonique en anglais est « HAVE + JUST + V-EN » se transforme en « BE + AFTER + V-ING » à maintes occasions comme

14. Flann O'Brien, The Third Policeman, Londres, MacGibbon \& Kee Ltd, 1967, p. 62 .

15. Ibid., p. 163.

16. R.P. = «Received Pronunciation», prononciation «reçue» ou standard consignée dans les dictionnaires de phonétique anglaise comme l'ouvrage reconnu par antonomase sous le nom de «Jones» (Cambridge University Press).

17. The Best of Myles, op. cit., p. 106.

18. Flann O'Brien, Faustus Kelly, in Stories and Plays, Londres, Hart-Davis, MacGibbon, 1973, p. 103-106

19. Flann O'Brien, At Swim-Two-Birds, op. cit., p. 23-24. Je souligne en caractères gras dans cet exemple et les suivants. 
en témoignent les quelques exemples suivants : «[...] he was after saying he was half-way down the road $[\ldots]^{20} »$, ou " [...] the Deluge was after coming again ${ }^{21} \gg$. Toujours sur le plan verbal, notons que l'auxiliaire du présent simple «do» se combine à l'aspect « $\mathrm{BE}+$ ING» en structure assertive, voire interrogative, ce qui serait fautif et pour le moins surprenant en anglais standard. On trouve ainsi des phrases comme : "What does any man be doing in a clump? ${ }^{22}$ ». Dans la pièce Thirst, on rencontre : "They do be bringin' handcarts up there ${ }^{23}$ » ou encore : "Does there be crows in the desert ? ${ }^{24} \gg$. Le present perfect disparaît des phrases où habituellement il marque la durée d'un événement entamé dans le passé et se prolongeant dans le présent. On trouve ainsi dans la transposition anglaise de The Poor Mouth de Patrick C. Power : "I'm two years waiting now ${ }^{25}$ ", au lieu de «I have been waiting for two years now", ou encore : " [she] was dying for twenty years in the bed $[\ldots]^{26} »$, au lieu de la formulation standard: "she had been dying for twenty years. "

Une autre caractéristique de l'anglais parlé en Irlande est l'interférence entre styles direct et indirect, là où on trouve habituellement « if » ou « whether » voire « that » suivis d'un schéma classique Sujet/Verbe/Objet dans la subordonnée. François Chevillet décrit très bien ce phénomène dans Les Variétés de l'anglais ${ }^{27}$. On en trouve un superbe exemple dans la bouche de l'oncle dans At Swim : "Do you think would the religious life appeal to you ${ }^{28}$ ? ».

La langue de notre corpus est donc typiquement irlandaise, sans parler du lexique d'origine gaélique, notamment pour les insultes : « pulthogue/polthogues », « gombeen men ${ }^{29}$, " (Cork) twisters »,

20. Flann O'Brien, The Third Policeman, Londres, op. cit., p. 84.

21. Myles, The Poor Mouth, op. cit., p. 101, je souligne.

22. Flann O'Brien, At Swim, op. cit., p. 119, je souligne.

23. Flann O'Brien, Thirst, in Stories and Plays, Londres, Hart-Davis, MacGibbon, 1973 , p. 84.

24. Ibid., p. 91.

25. MYLES, The Poor Mouth, op. cit., p. 79.

26. Ibid., p. 68.

27. François Chevillet, Les Variétés de l'anglais, Paris, Nathan Université, p. 136146.

28. Flann O'Brien, At Swim-Two-Birds, op. cit., p. 29.

29. Ibid., p. 112, voir aussi Faustus Kelly, in Stories and Plays, op. cit., qui en regorge, cf. p. 106, 112. 
«thullaramawns» «shut your gobbogue ${ }^{30} »$, etc. Cette langue sert notamment à la caractérisation. Elle apparaît souvent chez des personnages stéréotypés comme l'oncle du narrateur dans At Swim, les personnages caricaturaux et indéfinis de Shanahan et Lamont, ou encore les étranges policiers qui hantent les campagnes de The Third Policeman. L'irlandité de la prose d'O'Brien n'est donc pas lyrique ou idéalisée, elle produit un curieux effet de réel, parfois au service du fantastique comme dans The Third Policeman. Monique Gallagher souligne cette authenticité paradoxale quand elle nous dit : «O'Brien a toujours retenu, dans les qualités de l'art de Joyce, sa sensibilité au parler dublinois et la fidélité avec laquelle il le fait vivre dans son dialogue $[\ldots]^{31}$. »

Si O’Brien est fasciné par l'idiome dublinois, c'est parce que l'art de la parole est en soi captivant par son caractère vivant. Idiosyncrasies, clichés, particularismes constituent pour Flann O'Brien un terrain de jeux linguistiques privilégié, fertile en quiproquos, démystifications, ambiguïtés. L'irlandité de la prose de notre corpus participe donc de cette conscience linguistique aiguë. Elle constitue en grande partie le nerf du dialogue, et l'un des ressorts de l'humour. Cette irlandité implique des contours identitaires qui permettent précisément de jouer sur la définition d'une identité. Puisque les bornes sont posées, il est possible de les dépasser, transgresser, comme dans ce chapitre hyperbolique et satirique traitant des Gaeligores dans The Poor Mouth ${ }^{32}$, ou la racine « gael » est répétée près de 115 fois :

"There is nothing in this life so nice and so Gaelic as truly true Gaelic Gaels who speak in true Gaelic Gaelic about the truly Gaelic language. I hereby declare this feis to be Gaelically open! Up the Gaels! Long live the Gaelic tongue ${ }^{33}$ ! »

Le nationalisme est donc bien présent dans notre corpus mais pas en tant que simple credo politique, plutôt comme repoussoir dialectique et véritable révélateur du carcan idéologique imposé à la langue.

30. The Best of Myles, op. cit., p. 92.

31. M. GallaGHeR, op. cit., p. 156-157.

32. MyLes, The Poor Mouth, op. cit., p. 46-61.

33. Ibid., p. 54-55. 


\section{Ambiguïté et nationalisme}

On le voit, le thème du nationalisme chez O'Brien est un sujet dont l'ironie et la relativisation ne sont jamais très éloignées. Prenons par exemple cette phrase du narrateur d'At Swim :

«We talked together in a polished manner, utilizing with frequency words from the French language, discussing the primacy of America and Ireland in contemporary letters and commenting on the inferior work produced by writers of the English nationality ${ }^{34} . »$

Le lecteur inattentif pourrait se dire qu'il y a là une revanche prise contre l'ancienne puissance coloniale britannique. L'Irlande dominerait ainsi l'Angleterre sur le terrain de la littérature et des idées. Or le style est périphrastique («words from the French language», «writers of the English nationality», au lieu de «French words» ou «English writers»), pompeux («utilizing with frequency words from the French language») et ironique une fois mis en regard du contexte. En effet, la politesse et le raffinement dont il est question ici : «in a polished manner» semblent niés par la suite immédiate des événements :

«The Holy Name was often taken, I do not recollect with what advertence. Brinsley, whose education and maintenance was a charge on the rates of his native county - the product of a farthing in the pound applied for the purpose of enabling necessitous boys of promising intellect to enjoy the benefits of university learning - Brinsley said that he was prepared to give myself and Donaghy a pint of stout apiece, explaining that he had recently been paid ${ }^{35} . »$

Les périphrases interminables et le style ampoulé ne doivent pas tromper, les étudiants en question ne discourent pas posément de littérature, ils poussent des jurons ( The Holy Name was often taken») et se soucient d'alcool dont la consommation est payée par les contribuables du jeune État Libre d'Irlande. Si nous replaçons les

34. Flann O'Brien, At Swim-Two-Birds, op. cit., p. 45.

35. Ibid., p. 45. 
propos initiaux en contexte, on a donc un groupe d'étudiants fanfarons et pédants qui glorifient l'Irlande tout en dilapidant leurs bourses en buvant des bières. Du reste dans sa rubrique, O'Brien démontre son scepticisme à l'égard du système universitaire irlandais : «I sincerely believe that if University education were universally available and availed of, the country would collapse in one generation ${ }^{36}$. $\gg$ Il y a donc déflation de tout nationalisme éventuel par l'ironie manifeste du passage. D'ailleurs, si l'on se réfère à l'incipit du roman, les deux auteurs cités sont James Joyce (dont le poids sur la création littéraire irlandaise était jugé excessif par O'Brien lui-même) et Aldous Huxley, «the eminent English writer ${ }^{37} »$. La prétendue supériorité littéraire irlandaise perd donc en crédibilité. De même, la notion de «Home Rule» illustrée dans The Third Policeman par l'hystérie collective de la foule vindicative, est-elle ouvertement critiquée ${ }^{38}$. Dans At Swim, la ligue gaélique si typique de l'enthousiasme souvent mécanique du «Celtic Revival» pour O’Brien apparaît étroite d'esprit, conservatrice, pro-cléricale, fermée à tout ce qui n'est pas irlandais. La polémique sur la valse montre le ridicule vétilleux que peut incarner ce nationalisme :

«But after all a ceilidhe is not the place for it, that's all. $\boldsymbol{A}$ ceilidhe is a ceilidhe. I mean, we have our own. We have plenty of our own dances without crossing the road to borrow what we can't wear. [...] The Gaelic League is opposed to the old-time waltz, said Mr Corcoran. So are the clergy ${ }^{39}$. »

La tautologie domine, "A ceilidhe is a ceilidhe », image de cercle vicieux, d'esprit obtus. Mais c'est The Poor Mouth qui dénonce avec le plus de verve satirique les excès bornés du milieu culturel nationaliste irlandais. O'Brien brocarde les esprits à l'origine de la création du mythe de la nation irlandaise éternelle, pure, panceltique. L'Irlande du kilt (écossais) et des « gaeligores » est ainsi ridiculisée par O’Brien, comme en témoigne ce passage caustique :

36. Extrait tiré de la rubrique «Cruiskeen Lawn», The Irish Times, 2 mars 1966, soit moins d'un mois avant le décès de Myles.

37. Flann O'Brien, At Swim-Two-Birds, op. cit., p. 11.

38. Flann O'Brien, The Third Policeman, op. cit., p. 165.

39. Flann O'Brien, At Swim-Two-Birds, op. cit., p. 133-134, je souligne. 
«Crowds came from Dublin and Galway city, all with respectable, well-made clothes on them; an occasional fellow without any breeches on him but wearing a lady's underskirt instead. It was stated that such as he wore Gaelic costume and, if this was correct, what a peculiar change came as a result of a few Gaelic words in your head! There were men present wearing a simple unornamented dress -these I thought, had little Gaelic; others had such nobility, style and elegance in their feminine attire that it was evident that their Gaelic was fluent. I felt quite ashamed that there was not even one true Gael among us in Corkadoragha ${ }^{40}$.»

Ici le malentendu est total. La culture gaélique officielle des cercles celtiques urbains est tellement éloignée de la réalité du quotidien dans le Gaelacht que personne ne peut s'y identifier. Le narrateur est stupéfait de voir des hommes affublés de jupes. L'ironie est manifeste. La vision de l'irlandité présentée par les Gaeligores est reconstruite, artificielle, mythologique, erronée et procède d'une lecture très biaisée et partiale de l'histoire des Celtes. Les mêmes préjugés sur la langue gaélique sont vigoureusement dénoncés dans la scène qui clôt le chapitre 3 , où un Gaeligore (excès de la satire oblige) est admiratif face aux grognements d'un porc dans la pénombre d'un pub. Car pour ce Gaeligore fanatique et collecteur d'échantillons linguistiques:

" [...] good Gaelic is difficult but [...] the best Gaelic of all is well-nigh unintelligible. [...]

I do not know whether it was Gaelic or English or a strange irregular dialect which was in the old speech which the gentleman collected from among us here in Corkadoragha but it is certain that whatever was uttered that night, came from our rambling pig ${ }^{41}$.»

Finalement, cette idéologie étriquée est à l'image du «sea-cat» représenté page 77 et rappelant facétieusement la forme de l'île d'Irlande : monstrueuse.

40. Myles, The Poor Mouth, op. cit., p. 51-52.

41. Ibid., p. 44-45. 


\section{The Old-Fellow looked closely} at the picture* and a shadow crept over his visage.

- If that's how it is, son, said he fearfully, it's good news that you're alive today and in your health among us. What you met last night was the Sea-cat!

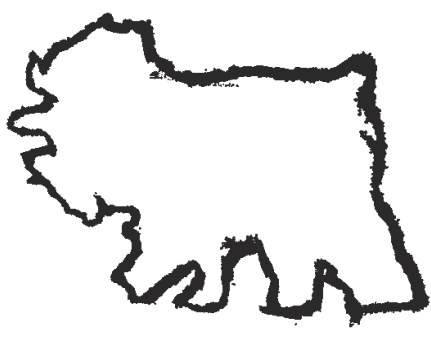
The Sea-cat!!

* The good reader will kindly notice the close resemblance between the Sea-cat, as delineated by $0^{\prime}$ Coonassa, and the pleassant little land which is our own. Many things in life are unintelligible to us but it is not without importance that the Sea-cat and Ireland bear the same shape and that both have all the same bad destiny, hard times and ill-luck attending on them which have come upon us.

Ainsi, l'Irlande et sa quintessence identitaire ${ }^{42}$, à savoir le gaélique, se trouvent traitées avec une ironie réflexive. Monstruosité cartographique se met alors à rimer avec monstruosité lexicographique.

\section{Gaélique chaotique : une langue impossible et proliférante?}

Ce qui sous-tend The Poor Mouth et un nombre conséquent de chroniques de Myles na gCopaleen est la dimension excessive, effrayante du gaélique. En effet, en sus du sens tautologique du terme monstrueux (« qui est propre à un monstre »), le Robert 1 nous

42. En effet l'irlandais est selon la constitution (qu'il s'agisse du texte de 1922 ou 1937) la première langue officielle devant l'anglais. Paradoxe saisissant dans un pays celtique où la langue la mieux et la plus utilisée demeure malgré tout l'anglais. O'Brien développe un point de vue à la fois ironique et convaincant sur ce thème constitutionnel dans Further Cuttings from Cruiskeen Lawn (Kevin O’Nolan, dir.), Londres, Hart-Davis, MacGibbon, 1976, p. 137. Citons : «I regret to announce that the Constitution, your ultimate and fundamental statement of your Irish identity and destiny, is an unconscionably careless document. Some of the English is bad and most of the Irish is disgracefully bad. More, the two languages frequently express dissimilar and mutually repugnant meanings in stating what purports to be the same Article.» Quand on songe que cette chronique a été publiée en première page du vénérable Irish Times, on comprend la portée iconoclaste de la pensée d'O'Brien. 
offre en guise de définition plus précise ceci : «qui est d'une taille, d'une intensité prodigieuse et insolite» ou encore : "qui choque extrêmement la raison, la morale». O'Brien nous laisse entrevoir une langue improbable, irrationnelle, une langue proliférante jusqu'au non-sens. Ses attaques visent alors l'un des gardiens du temple lexicographique : Dinneen, qui est un peu à l'irlandais ce que Littré fut au français au XIX ${ }^{\mathrm{e}}$ siècle. Et Dinneen ${ }^{43}$ sort de l'épreuve passablement égratigné. La figure singulière de Pádraig Ó Duinnín offre même par moments une certaine résonnance avec celle abracadrante du savant fou de Selby dans The Third Policeman. Et si l'on examine le lien entre ces deux figures, on s'aperçoit que c'est bien le rapport au langage, erratique en diable quant au réel, qui les réunit. Ainsi : "De Selby has some interesting things to say on the subject of houses. [...] Elsewhere he defines a house as a 'large coffin', a 'warren', and 'a box' ${ }^{44}$.»

Qu'est-ce donc qu'une maison? De Selby répond par une suite de métaphores déroutantes : un large cercueil, une garenne, une boîte. Le langage devient fuyant, imagé et, du même coup, inadéquat à rendre la réalité de façon rigoureuse. Mais n'est-ce pas là précisément l'essence de la langue irlandaise selon Dinneen et ce qu'en dit O'Brien?

«The Irish lexicographer, Dinneen, considered in vacuo is, heaven knows, funny enough. He just keeps standing on his head, denying stoutly that piléar means bullet and asserting that it means 'an inert thing or person'. Nothing stumps him. He will promise the sun moon and stars to anybody who will catch him out. And well he may. Just take the sun, moon and stars for a moment. Sun, you say is grian. Not at all. Dinneen shouts that grian means 'the bottom (of a lake, well)'. You are a bit nettled and mutter that, anyway, gealach means moon. Wrong again. Gealach means 'the white circle in a slice of a half-boiled potato,

43. Le père Patrick Dinneen est né le 25 décembre 1860 (jour prédestiné pour un futur prêtre), à Rathmore et décédé le 29 septembre 1934 à Dublin. On se souvient surtout de lui pour son Irish-English Dictionary publié d'abord en 1904 pour la Irish Texts Society puis constamment enrichi jusqu'à sa mort.

44. Flann O'Brien, The Third Policeman, op. cit., p. 22. 
turnip, etc.' In a bored voice he adds that réalta ${ }^{45}$ (of course) means 'a mark on the forehead of a beast'. Most remarkable man. Eclectic I think is the word.

That of course, is why I no longer write Irish. No damn fear. I didn't come down in the last shower. Call me a bit fastidious if you like but I like to have some idea of what I'm writing. Libel, you know. One must be careful. If I write in Irish what I conceive to be 'Last Tuesday was very wet,' I like to feel reasonably sure that what I've written does not in fact mean 'Mr So-and-So is a thief and a drunkard ${ }^{46}$.»

Le style dans ce passage est satirique, faisant de Dinneen un doux dingue qui divague et de l'irlandais une langue obscure impropre à désigner les éléments les plus habituels du quotidien comme la lune ou le soleil. On pourrait, en effet, se contenter de l'interprétation épigraphique dans d'autres contextes, mais O'Brien reprend ce thème de l'inadéquation de l'irlandais à faire sens et à transcrire le réel trop souvent pour que l'on balaie l'idée qu'effectivement sa vision de l'irlandais révèle son caractère digressif, insaisissable, parfois aberrant. Nous avons déjà fait référence à l'inintelligibilité du «bon » gaélique qui, si l'on croit les souvenirs comiques de Bonaparte O'Coonassa ${ }^{47}$, ressemble aux grognements d'un cochon. La même idée de grognement incompréhensible ouvert à toutes les exégèses possibles se retrouve développée dans The Best of Myles avec une nouvelle fois pour cible Dinneen, rappelant incidemment de Selby et son Codex ${ }^{48}$, également indéchiffrable : «The signal distinction of the manuscript is that not one word of the writing is legible ${ }^{49}$. 》 On voit donc s'insinuer de façon récurrente un thème inquiétant et drôle chez O'Brien, celui de la disjonction radicale entre le réel d'une part et sa représentation d'autre part, problématique chère à un philosophe français contemporain comme Clément Rosset qui identifie une idiotie du réel ${ }^{50}$. Ainsi, il y a possibilité pour qu'une langue, et entre toutes le gaélique, ne dise rien de clair. De même, l'hypothèse

45. Réalta $=$ étoile.

46. The Best of Myles, op. cit., p. 276-277.

47. Myles, The Poor Mouth, op. cit., p. 44.

48. Flann O'Brien, The Third Policeman, op. cit., p. 150.

49. Flann O'Brien, The Third Policeman, op. cit., p. 150.

50. Clément Rosset, Le Réel Traité de l'idiotie, Paris, les Éditions de Minuit, 1977. 
qu'une écriture ne signifie absolument rien d'objectif ou sensé et soit donc littéralement indéchiffrable est à prendre sérieusement en considération. Le réel demeure in fine impénétrable, irreprésentable en dépit de la conscience humaine. De ce fait potentiellement universel, O'Brien fait un trait typique - et comique - de l'irlandais et ce par un biais inattendu : sa richesse polysémique. Les mots en gaélique veulent dire tellement de choses qu'ils finissent par ne plus rien signifier, en un phénomène de prolifération suivi de saturation puis de disparition du sens précis. Dans l'extrait suivant, O'Brien pousse ce raisonnement jusqu'au non-sens, dans un crescendo de détails minutieux. De manière très irlandaise, par l'emploi superfétatoire de l'article défini the, la chronique qui nous intéresse s'intitule : «THE GAELIC». S'agit-il de la seule langue gaélique désignée par un irlandisme ? Des celtes (« Gaels ») en général ? des choses gaéliques en général ? Ces différentes interprétations ne sont pas à exclure. Ce qui est à noter c'est précisément cette réflexivité ambiguë du texte, cette conscience ironique de soi.

" There is scarcely a single word in the Irish (barring, possibly, Sasanach) that is simple and explicit. Apart from words with endless shades of cognate meaning, there are many with so complete a spectrum of graduated ambiguity that each of them can be made to express two directly contrary meanings, as well as a plethora of intermediate concepts that have no bearing on either. And all this strictly within the linguistic field. Superimpose on all that the miasma of ironic usage, poetic licence, oxymoron, plamás $^{51}$, Celtic evasion, Irish bullery and Paddy Whackery, and it is a safe bet that you will find yourself very far from home. Here is an example copied from Dinneen and from more authentic sources known only to my little self $[\ldots . .]^{52}$. »

Un détail ici révélateur et ironique se trouve dans la mention de «sasanach» (= saxon, anglais, à rapprocher du breton saozneg). L'Irlandais ne comprend rien de limpide, rien de simple, rien d'explicite à l'exception notable de l'Autre c'est à dire le Saxon, celui d'en face, image supplémentaire d'une langue doublement vide qui tire sa

51. Plamas : flagornerie, flatterie.

52. The Best of Myles, op. cit., p. 278. 
substance d'une opposition dialectique paradoxale. Sans Saxons pas d'Irlandais, mais impossible de définir en soi l'Irlandais. On trouve le même procédé à la mention des mots pour le moins curieux sous la plume d'un auteur de littérature : «the miasma of ironic usage, poetic licence, oxymoron », littéralement : " les miasmes de l'ironie, de la licence poétique et de l'oxymore ». O'Brien déconstruit son propos au fur et à mesure qu'il le construit car précisément, son texte est ironique, métaphorique et donc poétique, empli de licences poétiques et d'antiphrases. "Miasma» est un terme très fort et péjoratif qui s'applique justement au texte en construction qui vient parasiter, déranger, réorganiser les référents idéologiques existants. La litanie qui s'ensuit est stupéfiante tant le contenu sémantique suscité par le mot très court «cur» semble tiré par les cheveux. Le comique du non-sens couronne le comique de la disproportion : « cur » désigne la dentition d'un leprochaun, le furoncle d'un héron, un biscuit à mouton, le gonflage d'un boyau de lièvre avec une pompe à vélo, le bruit fait dans une maison vide par une personne non autorisée etc. La règle de la réflexivité ironique s'impose, il s'agit de fadaises, d'histoires à dormir debout typiquement irlandaises, c'est-à-dire précisément des «Irish bullery» et «Paddy Whackery» fallacieusement dénoncées. L'énumération va jusqu'à l'épuisement du procédé :

«Cur, g. curtha and cuirthe, m. -act of putting, sending, sowing, raining, discussing, burying, vomiting, hammering into the ground, throwing through the air, rejecting, shooting, the setting or clamp in a rick of turf, selling, addressing, the crown of cast-iron buttons which have been made bright by contact with cliff-faces, the stench of congealing badgers'suet, the luminance of glue-lice, a noise made in an empty house by an unauthorized person, a heron's boil, a leprachaun's denture, a sheep-biscuit, the act of inflating hare's offal with a bicycle pump, a leak in a spirit level, the whine of a sewage farm windmill, a corncrake's clapper, the scum on the eye of a senile ram, a dustman's dumpling, a beetle's faggot, the act of loading every rift with ore, a dumbman's curse, a blasket, a 'kur', a fiddler's occupational disease, a fairy godmother's father, a hawk's vertigo, the art of predicting past events, a wooden coat, a custard-mincer, a blue-bottle's 'farm', a gravy flask, a timber-mine, a toy craw, a porridge-mill, a fair-day donnybrook with nothing barred, a stoat's stomach-pump, a broken- 
But what is the use? One could go on and on without reaching anywhere in particular ${ }^{53} . »$

Quarante-quatre définitions sont ainsi suggérées. L'effet d'accumulation et de surenchère dans la gradation est marqué par une conscience réfléchie de sa gratuité : «But what is the use? One could go on and on without reaching anywhere in particular.» $\mathrm{La}$ conclusion du passage constitue à cet égard un sommet de la raillerie :

«The plight of the the English speaker with his wretched box of 400 vocal beads may be imagined when I say that a really good Irish speaker would blurt out the whole 400 in one cosmic grunt. In Donegal there are native speakers who know so many million words that it is a matter of pride with them never to use the same word twice in a life-time. Their life (not to say their language) becomes very complex at the century mark ${ }^{54}$.»

Exagération (400 lexèmes prononcés simultanément ne constituent pas une réalité envisageable sérieusement, même dans le Gaeltacht), métaphore cocasse et hyperbolique par l'adjectivation grandiloquente (" one cosmic grunt»), euphémisme et exagération combinées - «Their life (not to say their language) becomes very complex at the century mark»-, nous amènent à une conception du langage purement idiosyncrasique. L'idée d'un locuteur ne prononçant jamais deux fois le même mot par fierté est absurde et drôle par son excès caricatural et paradoxal. L'anecdote joue sur le cliché du fier Irlandais poète créateur tout en soulignant le fait qu'un langage collant trop à la réalité du locuteur, en d'autres termes un langage fait d'hapax legomena, serait ingérable, incompréhensible. On connaît l'aversion d'O'Brien à l'encontre des clichés, et son "Catéchisme du cliché» l'a institué en véritable pourfendeur du prêt-à-penser. Mais son texte lui-même, si l'on adopte un mouvement derridéen de déconstruction, nous rappelle qu'il est nécessaire d'utiliser un fonds commun d'idées déjà conçues et partagées pour nous faire nous comprendre, sous peine de prendre le risque de tomber dans ce que Rosset appelle l'idiotie du réel ${ }^{55}$; à savoir son caractère tellement

53. Ibid., p. 278-279. «cur» renvoie au verbe anglais «put».

54. Ibid., p. 279.

55. Cf. Clément Rosset, Le Réel Traité de l'idiotie, op. cit. 
étrange, singulier et incommunicable que le locuteur adopte la figure de l'«idiot». La racine étymologique de ce mot («idiot») est bien identique à celle d' «idiosyncrasie» (du grec idiosugkrasia : tempérament particulier, trop particulier ici sans doute). On peut donc dire que l'attitude de Flann O'Brien quant à l'irlandité est des plus complexes, car réflexive et ironique. Moquant les excès lyriques et subjectifs d'un Patrick Dinneen, il joue plus volontiers sur les registres du pastiche, de la méfiance/défiance ludiques et de la déconstruction, ce faisant, sa démarche est d'une ambiguïté remarquable car sa critique féroce de l'Irlande conduit plus que jamais à une surassertion sans commune mesure du particularisme irlandais. Sans doute cette dynamique contradictoire du texte est-elle due à la profonde spécularité des procédés stylistiques employés. O'Brien est donc ainsi un auteur typiquement irlandais sans tomber pour autant dans le piège de l'écriture identitaire militante, folklorisante ou monologique, à la manière de sa vision jubilatoire de Saint Patrick.

"'Two St. Patricks? We have four of the buggers in our place and they'd make you sick with their shamrocks and shenanigans and bullshit ${ }^{56}$.»

56. Flann O’Brien, The Dalkey Archive, Londres, MacGibbon \& Kee, 1964, p. 35. 